\title{
Challenging Cases in Urothelial Cancer
}

\author{
Mark S. Soloway* \\ Urologic Oncology, Memorial Physician Group, Division of Urology, Memorial Healthcare System, \\ Aventura, FL, USA
}

Received/Accepted 11 December 2018

\section{CASE 11}

L.R. is a healthy appearing 83 year old woman that presented to a urologist upon referral from her primary care physician with a 6 month history of recurrent urinary tract infections and more recently some right flank discomfort. A CT scan of the abdomen and pelvis revealed moderate right hydronephrosis with a point of obstruction near or at the bladder. The left upper tract was normal. Images of the bladder indicated masses consistent with bladder cancer.

The patient never smoked cigarettes. She has not had any major surgery. She has not had gross hematuria and has no voiding complaints.

Her chest $\mathrm{x}$ ray was normal. All of her lab work including a BUN and creatinine were normal.

The urologist proceeded with a cystoscopy which revealed multifocal papillary bladder tumors. The tumors were primarily located on the right side of the bladder. A transurethral resection of some tumor was performed. The patient had bilateral percutaneous nephrostomy tubes placed. It is unclear why a left percutaneous nephrostomy was placed. The pathology report indicated that the tumor was high grade and invaded the lamina propria (HG T1). She was advised that a cystectomy would be her best option.

I saw the patient and suggested that a re TUR BT was appropriate in order to see whether bladder preservation was a possible initial alternative. She was taken to the operating room Dec. 2018. A bimanual examination was normal. On cystoscopy using a 70 and then a 30 degree lens I found that the

\footnotetext{
*Correspondence to: Mark S. Soloway, MD, Chief, Urologic Oncology, Memorial Physician Group, Division of Urology, Memorial Healthcare System, Aventura, FL, USA. E-mail: msso loway@yahoo.com.
}

right side of her bladder was covered with papillary bladder tumors. The tumors extended along the entire right trigone and continued up the lateral wall and posterior and also inferiorly to the right bladder neck from 6 to 12 o'clock. The left side of the bladder was mostly tumor free. The pathology was HG Ta. There was no muscle in the specimen and due to cautery artifact the pathologist could not determine whether there was lamina propria invasion. Despite my attempt to obtain muscle in the specimen there was none to be found. I spent approximately $45 \mathrm{~min}$ utes performing the resection before stopping. There was minimal blood loss. I estimated I resected $80-$ $90 \%$ of the visible tumor.

In an effort to make her more comfortable I had the interventional radiologist perform bilateral nephrostograms and try to place a right $\mathrm{JJ}$ stent and if there was no obstruction on the left side remove the left nephrostomy. This was successful.

My initial impression when I met this woman was that given the extensive tumor (based on the operative report and CT images) and the right ureteral obstruction she would require a cystectomy. Having performed the second TUR BT I am not certain what is best.

There are several options: 1 . Proceed with a cystectomy/urinary diversion as this will almost certainly provide a cure and therefore eliminate further TURs which are likely; 2. Perform another TUR BT with the goal of more accurately establishing the tumor stage which is likely $\mathrm{Ta} / \mathrm{T} 1$ and give her an opportunity to avoid a urinary diversion and the morbidity of a RC; 3. Prescribe intravesical therapy first and then a re TUR BT.

We invite our readers to review and comment on the case and management by using the online comment section below the case: https://www.bladdercancer journal.com/challenging-cases 


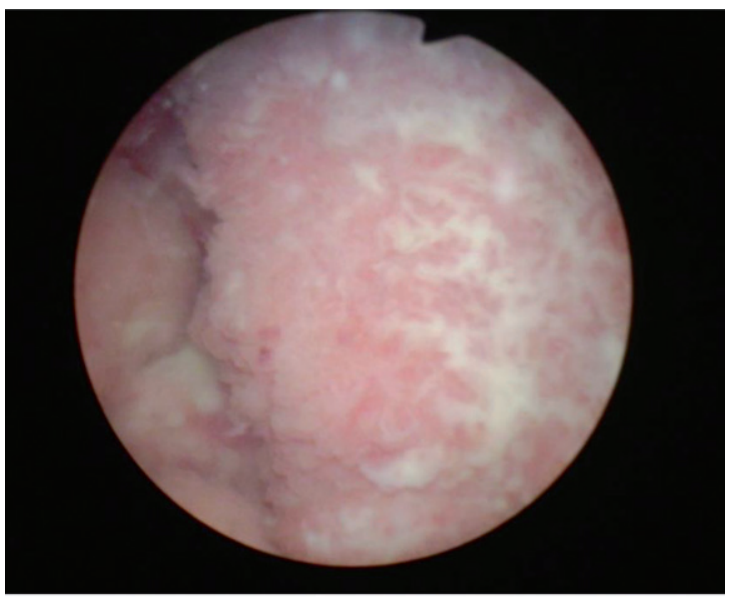

\section{CONFLICTS OF INTEREST}

Nothing to disclose.
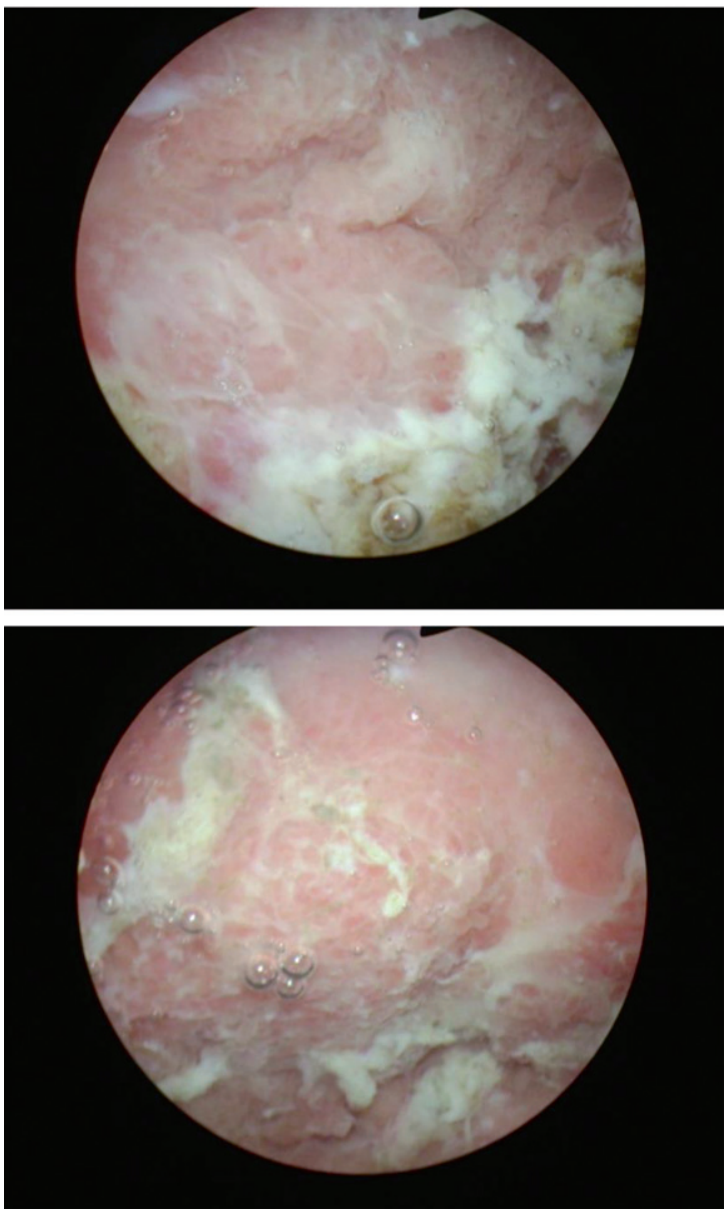

Fig. 1-3. Papillary high grade Ta urothelial cancer. Tumor occupies much of the right side of the bladder. 